\title{
Optimized search strategy for detecting scientifically strong studies on treatment through PubMed
}

\author{
Salvatore Corrao $\cdot$ Daniela Colomba $\cdot$ \\ Christiano Argano · Luigi Calvo • Rosario Scaglione • \\ Giuseppe Licata
}

Received: 30 December 2011/ Accepted: 1 March 2012/Published online: 17 March 2012

(c) SIMI 2012

\begin{abstract}
Our study was designed to optimize the search strategies based on the work of Haynes et al. for detecting randomized controlled trials (RCTs) through PubMed. In particular, we aimed to improve precision for broad and narrow searches on interventional studies. We used in addition to the string suggested by the Hedge Team the following: \{NOT ((animals [mh] NOT humans [mh]) OR (review [pt] OR meta-analysis [pt])) \} and tested its effectiveness. The search was carried out on a year's worth of articles from the PubMed database. We analyzed 35,590 bibliographic citations about four relevant major topics in internal medicine (hypertension, diabetes, heart failure, and hepatitis). Precision, percentage gain between the Hedge Team search strategies and the new one were computed and reported in the text. Moreover, a pooled analysis was carried out in terms of absolute precision difference. We observed better precision for both broad and narrow searches. However, effective gain resulted only for broad searches. In this case, bibliographic citation recall effectively reduced $(-24$ to $-35 \%$ retrieved citation with a
\end{abstract}

S. Corrao $(\bowtie) \cdot$ D. Colomba · L. Calvo · G. Licata Centre of Research for Effectiveness and Appropriateness in Medicine (C.R.E.A.M), Dipartimento Biomedico di Medicina Interna e Specialistica (DiBiMIS), University of Palermo, Piazza delle Cliniche 2, 90127 Palermo, Italy

e-mail: s.corrao@tiscali.it

C. Argano

Divisione di Medicina Interna, Fondazione San

Raffaele-G.Giglio, Cefalù, Palermo, Italy

R. Scaglione

Laboratory of Clinical Epidemiology, Biostatistics and Knowledge Management, Dipartimento Biomedico di Medicina Interna e Specialistica (DiBiMIS), Università di Palermo, Piazza delle Cliniche 2, 90127 Palermo, Italy gain of 32-54\%) without loss of information. The search strategy improved broad searches regarding each of the four considered topics. We think this new search strategy, based on a previous work of the Hedge team, could be a step forward and can save some time by researchers.

Keywords PubMed - Medline · Databases .

Bibliographic · Information storage and retrieval · Therapy
Abbreviations
dp Publication date
pt Publication type
mh MeSH terms
sh MeSH subheading
tiab Title/abstract

\section{Introduction}

PubMed is a web-free search engine widely used by healthcare professionals. It was developed and maintained by the National Center for Biotechnology Information (NCBI) at the National Library of Medicine (NLM) located at the National Institutes of Health. It covers many fields of biomedical knowledge: medicine, nursing, dentistry, veterinary medicine, the health care system, and preclinical sciences. PubMed comprises more than 20 million bibliographic citations and abstracts from MEDLINE, life sciences journals, and online books. All the articles in PubMed Central (free-full text journals) are included. The search filters widely utilized in PubMed are based on the work of Haynes et al. [1] and have been updated with better performance [2] both for clinicians and researchers. However, the information retrieval can be very much time 
consuming because searches that identify all relevant information also may find irrelevant articles. Indeed, few users are trained in search techniques so that they run the risk of excessive recall that provides an unnecessary information overload.

Well-established strategies need to be further optimized to enhance effectiveness of search and to reduce the number of total retrieved citations without missing key studies.

In particular, this study concerned with searches on interventional studies, and was designed to optimize and test a modified search strategy for detecting randomized controlled trials (RCTs) through PubMed.

\section{Materials and methods}

Pubmed uses the MeSH (Medical Subject Headings) database that is the US National Library of Medicine's (NLM) controlled vocabulary used for indexing articles for MEDLINE. MeSH terminology provides a consistent way to retrieve information that may use different terminology for the same concepts, and is hierarchically organized by a tree (stem and leaf) structure. To retrieve studies on human beings the MeSH database includes the term "Humans". In the MeSH tree structure the term "Humans" is hierarchically subordinate to "Animals" (Fig. 1). The search using "Humans" as MeSH term will retrieve only indexed citations, so that there will be loss of both not yet indexed citations and citations that will be never indexed in MEDLINE. On the other hand, the search results using the MeSH term "Animals" will include all the citations indexed by the term "Animals," and all the terms hierarchically subordinate (PubMed automatically explodes the $\mathrm{MeSH}$ term using all the subordinate terms). There are seven terms between "Animals" and "Humans" (Fig. 1). Thus, the search using "Animals" (as MeSH term) will retrieve all the citations, most of whom will not concern human beings. Moreover, excluding the term "Animals" will exclude all the bibliographic citations indexed with the term "Humans".

On the contrary, a search string as follows "NOT (animals [mh] NOT humans [mh])" can exclude all the citations regarding articles indexed with "Animals" (MeSH term) that are not also indexed with "Humans" (MeSH term). Consequently, this search string will exclude all the citations regarding studies on animals that are not about human beings, reducing the number of retrieved citations. Moreover, if all the meta-analyses and reviews are excluded from the search results there will be further reduction in the number of retrieved citations to detect relevant ones. Indeed, if this kind of publication type is searched the tag [pt] can be used by the following search strings: metaanalysis [pt] OR review [pt]. In this way, meta-analyses and reviews can be separately analyzed.

Finally, the complete string proposed and tested was "NOT ((animals [Mesh] NOT humans [Mesh]) OR (review [pt] OR meta-analysis [pt]))" and joined to Haynes' search strings (http://www.ncbi.nlm.nih.gov/entrez/query/static/ clinicaltable.html) for both broad searches and narrow searches. Moreover, we tested the new string joined to each of four relevant topics considered of broad interest in
Fig. 1 Section of the MeSH tree structure: relationship between the term Animals and Humans (in boldface) is shown according to MeSH hierarchy

\author{
All MeSH Categories \\ Organisms Category \\ Eukaryota
}

Animals

Chordata

Vertebrates

Mammals

Primates

Haplorhini

Catarrhini

Hominidae

Humans 
internal medicine: hypertension, hepatitis, diabetes, and heart failure. A narrow and a broad search were performed for each topic, and the bibliographic citations retrieved were analyzed.

The search was performed on 2 March 2009 at 11:39 a.m. and the year considered was 2006. This particular year was chosen for its consistency and stability regarding both the NLM indexing process and the bibliographic citation loading process. In the Appendix we describe the search strategy in the final version. We considered RCTs from all clinical studies that met the following criteria:

1. at least a control group and an intervention group;

2. assignment of intervention using randomization;

3. declared endpoints.

No critical evaluation of trial quality was made because it is outside the objective of the Hedge Team and, consequently, of our study.

Two observers (DC, CA) have manually evaluated all the retrieved citations and formally checked for RCTs. Another person (SC) reviewed the entire process.

Statistical analysis

Precision was computed. Precision gain was computed as relative (percentage) or absolute differences. $95 \%$ confidence intervals $(95 \% \mathrm{CI})$ were computed for precision, percentages and absolute risk differences. The pooling was performed for broad and narrow searches, and the PubMed Clinical Query was compared with the search strategy modified.

Fixed effects (Mantel-Haenszel, Greenland-Robins) and Random effects (DerSimonian-Laird) techniques were used. Chi-square was computed. Non-combinability (heterogeneity) was reckoned by the Cochran's $Q$ and the moment-based estimate of between different topic variance. The random effects model was chosen if heterogeneity resulted in a statistically significant (alpha error $<0.05$ ).

\section{Results}

Overall, 35,590 bibliographic citations were included in the analysis. Our findings are shown in Table 1. Generally, the proposed search strategy increased the precision. The percentage gain ranged from 31.7 to $54.1 \%$ for broad searches and from 1.7 to $6.9 \%$ for narrow searches. Precision increased from 0.10 to 0.15 for hypertension, from 0.11 to 0.17 for diabetes, from 0.11 to 0.16 for heart failure, and from 0.08 to 0.10 for hepatitis regarding broad searches, with a reduction of recalled citations ( -24 to $-35 \%)$.

Regarding narrow searches, precision increased from 0.92 to 0.96 for hypertension, from 0.92 to 0.95 for diabetes, from 0.87 to 0.93 for heart failure and from 0.95 to 0.97 for hepatitis.

The pooled analysis for comparisons between broad and narrow searches in terms of absolute precision differences is shown in Fig. 2.

The pooled absolute precision difference resulted in 0.047 (95\% CI 0.033-0.061) for broad searches and 0.036 (95\% CI 0.020-0.052) for narrow searches. However, the hepatitis topic showed the lowest precision gain, which was not significant when narrow searches were performed.

Finally, increase of precision involved no loss in the total retrieved RCTs.

Table 1 Comparisons between Haynes' search strategy and the modified search one

\begin{tabular}{|c|c|c|c|c|c|c|c|}
\hline \multirow[t]{2}{*}{ Topics } & \multicolumn{3}{|c|}{ Haynes' strategy } & \multicolumn{3}{|c|}{ Modified strategy } & \multirow[t]{2}{*}{ Gain $(\%)$} \\
\hline & TRCs & RCTs & $\operatorname{Pr}(95 \%$ IC $)$ & TRCs & RCTs & $\operatorname{Pr}(95 \%$ IC $)$ & \\
\hline \multicolumn{8}{|l|}{ Broad search } \\
\hline Hypertension & 5,491 & 549 & $0.10(0.09-0.11)$ & 3,564 & 549 & $0.15(0.14-0.17)$ & $54.1(38.0-72.1)$ \\
\hline Diabetes & 7,252 & 808 & $0.11(0.10-0.12)$ & 4,855 & 808 & $0.17(0.16-0.18)$ & $49.8(36.5-63.5)$ \\
\hline Heart Failure & 3,112 & 344 & $0.11(0.10-0.12)$ & 2,096 & 344 & $0.16(0.15-0.18)$ & $48.5(29.2-70.6)$ \\
\hline Hepatitis & 2,890 & 228 & $0.08(0.07-0.09)$ & 2,195 & 228 & $0.10(0.09-0.12)$ & $31.7(10.6-56.8)$ \\
\hline \multicolumn{8}{|l|}{ Narrow search } \\
\hline Hypertension & 599 & 550 & $0.92(0.89-0.94)$ & 571 & 550 & $0.96(0.94-0.98)$ & $4.9(2.0-8.2)$ \\
\hline Diabetes & 882 & 810 & $0.92(0.89-0.94)$ & 854 & 810 & $0.95(0.93-0.96)$ & $3.3(0.7-6.0)$ \\
\hline Heart Failure & 388 & 339 & $0.87(0.84-0.90)$ & 363 & 339 & $0.93(0.90-0.96)$ & $6.9(2.1-12.3)$ \\
\hline Hepatitis & 241 & 230 & $0.95(0.92-0.98)$ & 237 & 230 & $0.97(0.94-0.99)$ & $1.7(-2.0-5.9)$ \\
\hline
\end{tabular}

Precision is approximate at the second decimal value; gain (\%) is computed by precision values

TRCs total retrieved citations, RCTs number of recognized randomized controlled trials, $\operatorname{Pr}$ precision, $C I$ confidence interval, gain (\%) relative gain of the modified search strategy in percentage 
Fig. 2 Absolute precision difference pooling analysis: Haynes'search strategies versus a modified search strategy (Random effect model was used for heterogeneity)

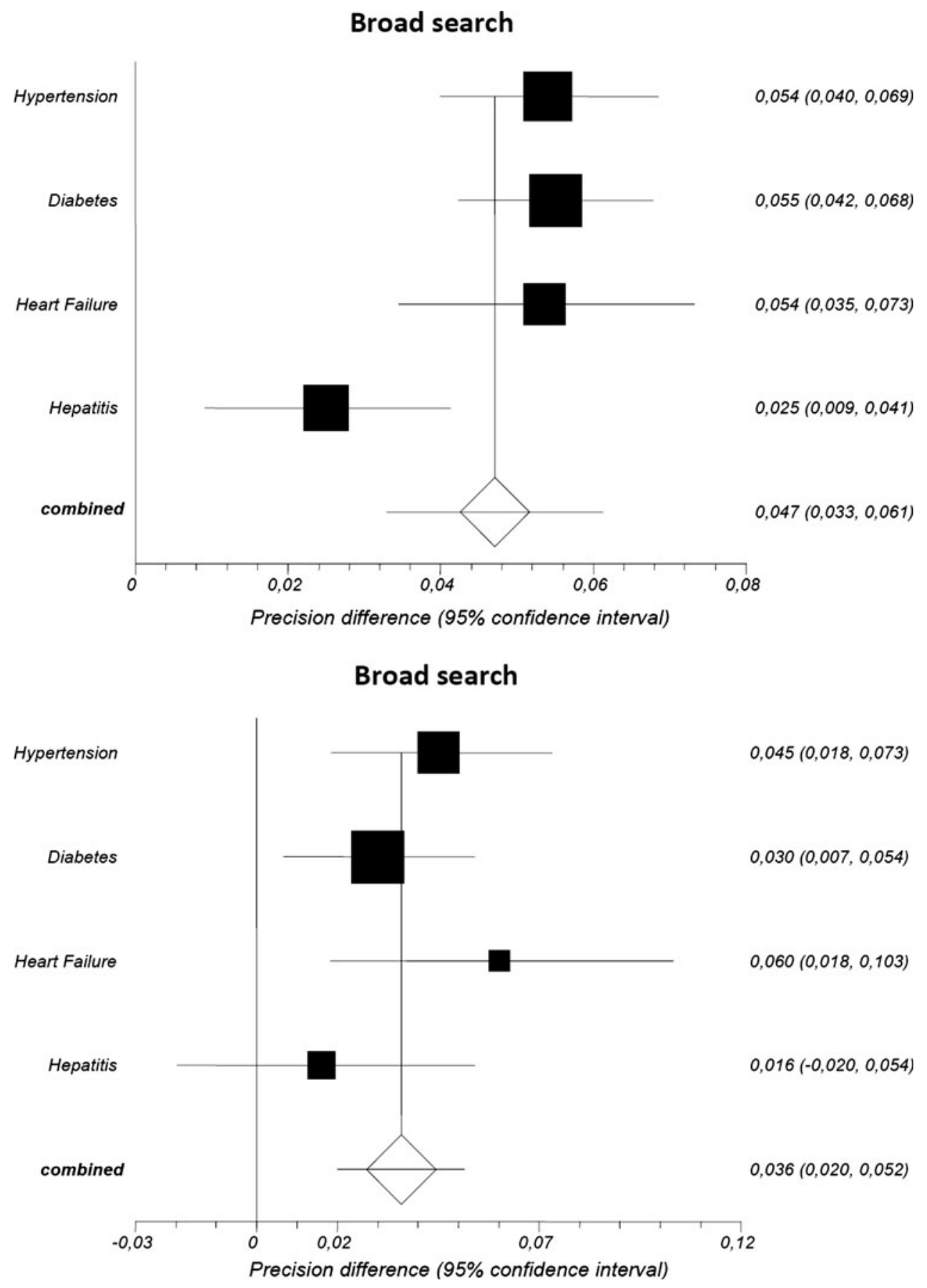

\section{Discussion}

The biomedical databases like MEDLINE are important sources of evidence in medical practice, and PubMed is one of the most relevant and freely available search engines of bibliographic citations. Several approaches have been developed to improve retrieval of studies clinically relevant and scientifically sound from similar bibliographic databases [3-10].

Haynes et al. [1] developed separate strategies for different purposes: strategies with high sensitivity for comprehensive searching and strategies with high precision for more focused searching. Robinson and Dickersin [3] tested a revised search strategy of all three phases of the Cochrane Highly Sensitive Search Strategy (HSSS) for accessing MEDLINE through both Ovid and PubMed. Minor modifications to the first two phases of the HSSS were put forth by Lefebvre and Clarke [9], and later tested by Zhang et al. in 2006 [10].

Our interest in the optimization of search filters motivated our previous work [11] cited by McKibbon et al. 
[12], who, recently, pointed out that our filter has virtually the same sensitivity, specificity, and precision as Haynes' specific filters. In addition, our filter showed a capability to retrieve more recent or non-indexed RCTs than Haynes' filters.

Nevertheless, enhancing effectiveness of searches and developing well-established search filters should be welcomed, and we thought about developing a new PubMed search strategy for detecting RCTs to improve precision searching on intervention studies for broad and narrow searches.

Our string showed an enhanced precision, the total citations retrieved were reduced, while the RCT number was the same for both broad and narrow searches with reference to the four considered topics.

Our results show that precision generally increases, in particular, when broad searches are performed. On the contrary, the modified strategy did not show a relevant precision gain for narrow searches.

In conclusion, no relevant improvement was shown by a new search strategy for narrow (specific) searches, but the new search strategy can be utilized for broad (sensitive) searches.

We believe that our study based on Haynes' work may contribute to research in this field, suggesting a new search strategy for detecting RCTs through PubMed, allowing a more efficient recovery of citations yielding a corresponding time saving, in particular, when systematic research is needed. We hope that this finding will become a part of the evidence used by systematic reviewers and information specialists in making decisions on developing their search strategies for systematic reviews.

Conflict of interest None.

\section{Appendix—search strategies}

\author{
Narrow search \\ \# 1:"search term"1 AND 2006 [dp]. \\ \# 2: (randomized controlled trial [pt] OR (randomized \\ [tiab] AND controlled [tiab] AND trial [tiab])). \\ \# 3: \# 1 AND \# 2. \\ \# 4: \# 3 NOT ((animals [mh] NOT humans [mh]) OR \\ (review [pt] OR meta-analysis [pt])). \\ Broad search
}

\# 1: "search term" AND 2006 [dp].

\# 2: ((clinical [tiab] AND trial [tiab]) OR clinical trials [mh] OR clinical trial [pt] OR random*[tiab] OR random allocation [mh] OR therapeutic use [sh]).

\# 3: \# 1 AND \# 2.

\# 4: \# 3 NOT ((animals [mh] NOT humans [mh]) OR (review [pt] OR meta-analysis [pt])).

\section{References}

1. Haynes RB, Wilczymski N, McKibbon KA, Walker CJ, Sinclair JC (1994) Developing optimal search strategies for detecting clinically sound studies in MEDLINE. J Am Inform Assoc $1: 447-458$

2. Hedges Team, Haynes RB, McKibbon KA, Wilczynski NL, Walter SD, Werre SR (2005) Optimal search strategies for retrieving scientifically strong studies of treatment from MEDLINE: analytical survey. BMJ 330(7501):1179

3. Robinson KA, Dickersin K (2002) Development of a highly sensitive search strategy for the retrieval of reports of controlled trials using PubMed. Int J Epidemiol 31:150-153

4. Nwosu CR, Khan KS, Chien PF (1998) A two-term MEDLINE search strategy for identifying randomized trials in obstetrics and gynecology. Obstet Gynecol 91:618-622

5. Marson AG, Chadwick DW (1996) How easy are randomized controlled trials in epilepsy to find on MEDLINE? The sensitivity and precision of two MEDLINE searches. Epilepsia 37:377-380

6. Adams CE, Power A, Frederick K, LeFebvre C (1994) An investigation of the adequacy of MEDLINE searches for randomized controlled trials (RCTs) of the effects of mental health care. Psychol Med 24:741-748

7. Dumbrigue HB, Esquivel JF, Jones JS (2000) Assessment of MEDLINE search strategies for randomized controlled trials in prosthodontics. J Prosthodont 9:8-13

8. Jadad AR, McQuay HJ (1993) A high-yield strategy to identify randomized controlled trials for systematic reviews. Online $\mathrm{J}$ Curr Clin Trials No 33

9. LeFebvre C, Clarke MJ (2001) Identifying randomised trials. In: Egger M, Smith GD, Altman DG (eds) Systematic reviews in health care: meta-analysis in context. BMJ, London, pp 69-86

10. Li Zhang, Ajiferuke I, Sampson M (2006) Optimizing search strategies to identify randomized controlled trials in MEDLINE. BMC Med Res Methodol 6:23

11. Corrao S, Colomba D, Arnone S, Argano C, Di Chiara T, Scaglione R, Licata G (2006) Improving efficacy of PubMed clinical queries for retrieving scientifically strong studies on treatment. J Am Inform Assoc 13(5):485-487

12. Team Hedges, McKibbon KA, Wilczynski NL, Haynes RB (2009) Retrieving randomized controlled trials from MEDLINE: a comparison of 38 published search filters. Health Info Libr J 26(3):187-202

\footnotetext{
1 search term indicates one of the four topics considered (hypertension, diabetes, heart failure, and hepatitis). For each of them broad and narrow searches were performed.
} 